Araştırma Makalesi - Research Article

\title{
Genelleştirilmiş Sylvester Transpoz Matris Denkleminin Simetrik - Ters Simetrik Ayrışım Metodu ile Çözümü
}

\author{
Murat SARDUVAN ${ }^{1 *}$, Esra KAPLAN ${ }^{2}$
}

Geliş / Received: 06/01/2020

Revize / Revised: 20/04/2020

Kabul / Accepted: 24/05/2020

ÖZ

Bu çalışmada $\boldsymbol{A} \boldsymbol{x}=\boldsymbol{b}$ lineer denklem sisteminin çözümü için Simetrik -Ters Simetrik Ayrışım (SSS) metodu tanitıldi. Daha sonra

$\mathrm{AXB}+\mathrm{CXD}+\mathrm{EX}^{\mathrm{T}} \mathrm{F}=\mathrm{M}$

genelleştirilmiş Sylvester transpoz matris denkleminin çözümü bu metot kullanılarak ortaya konuldu. Son olarak SSS metodunun performansını resmeden sayısal bir örnek verildi.

Anahtar Kelimeler-SSS metodu, Genelleştirilmiş Sylvester transpoz matris denklemi, Kronecker çarpım, Matris normu, Spektral yarıçap.

\footnotetext{
1*Sorumlu yazar iletişim: msarduvan@sakarya.edu.tr (https://orcid.org/0000-0001-7049-8922)

Matematik Bölümü, Sakarya Üniversitesi, Esentepe Kampüsü, Serdivan, Sakarya

2İletişim: esra.kaplan2@ogr.sakarya.edu.tr (https://orcid.org/0000-0002-1872-1987)

Matematik EABD Lisansüstü Öğrencisi, Sakarya Üniversitesi, Esentepe Kampüsü, Serdivan, Sakarya
} 


\title{
On Solution of Generalized Sylvester Transpose Matrix Equation Using Symmetric - Skew Symmetric Splitting Method
}

\author{
ABSTRACT \\ In this study, Symmetric-Skew Symmetric Splitting (SSS) method is introduced to solve the system of linear \\ equations $\boldsymbol{A} \boldsymbol{x}=\boldsymbol{b}$. Then, the solution of \\ $\mathrm{AXB}+\mathrm{CXD}+\mathrm{EX}^{\mathrm{T}} \mathrm{F}=\mathrm{M}$ \\ generalized Sylvester transpose matrix equation is established by using this method. Lastly, an example is given \\ to illustrate the performance of the SSS method.
}

Keywords- SSS method, Generalized Sylvester transpose matrix equation, Kronecker product, Matrix norm, Spectral radius. 


\section{GİRIŞ}

$m$ ve $n$ pozitif tam sayılar olmak üzere, $\mathbb{R}^{m \times n}, \mathbb{C}^{m \times n}, \mathbb{R}^{n}$ ve $\mathbb{C}^{n}$, sirası ile, $m \times n$ boyutlu reel matrisler, $m \times n$ boyutlu kompleks matrisler, $n \times 1$ boyutlu reel vektörler ve $n \times 1$ boyutlu kompleks vektörler kümelerini göstermektedir. $A^{T}$ ve $A^{*}$ notasyonları ile herhangi bir $A \in \mathbb{C}^{m \times n}$ matrisinin, sırasıyla, transpozu ve eşlenik transpozu gösterilmektedir. $A \otimes B$ ile herhangi iki $A, B \in \mathbb{C}^{m \times n}$ matrislerinin Kronecker çarpımı gösterilmektedir.

Bir $A \in \mathbb{C}^{n \times n}$ matrisi için; eğer $A=A^{*}$ oluyorsa matrise hermityen, eğer $A=-A^{*}$ oluyorsa matrise ters hermityen matris denir. Bir $A \in \mathbb{C}^{n \times n}$ matrisi için eğer $A=A^{T}$ sağlanıyorsa $A$ matrisine simetrik matris denir. Bir simetrik $A \in \mathbb{R}^{n \times n}$ matrisi, eğer her $x \in \mathbb{R}^{n}$ sıfırdan farklı vektörü için $x^{T} A x>0$ koşulunu sağlıyorsa $A$ matrisine pozitif tanımlıdır denir. Bir $A \in \mathbb{C}^{n \times n}$ matrisi için eğer $M$ tersinir ise $A=M-N$ ifadesine, $A$ matrisinin bir ayrışımı denir. $A=M-N$ ayrışımı kullanılarak $M x^{(k+1)}=N x^{(k)}+b, k=0,1,2, \ldots$, şeklinde iteratif metotlar kullanılabilir. Böylece, $C=I-M^{-1} A=M^{-1} N$ matrisi bu yapıdaki $x^{(k)}$ için katsayılar matrisi olup iterasyon matrisi adını alır. Bir $A \in \mathbb{C}^{n \times n}$ matrisi çok büyük boyutlu ve elemanlarının çoğu ' 0 ' ise $A$ matrisine büyük seyrek matris denir. $\lambda_{1}, \lambda_{2}, \ldots, \lambda_{n}$ özdeğerlerine sahip herhangi bir $A \in \mathbb{C}^{n \times n}$ matrisi için $\rho(A)=\max _{1 \leq i \leq n}\left|\lambda_{i}\right|$ değerine $A$ matrisinin spektral yarıçapı denir.

\|\|$. \| \mid: \mathbb{C}^{n \times n} \rightarrow \mathbb{R}$ fonksiyonu, eğer $\forall A, B \in \mathbb{C}^{n \times n}$ için aşağıdaki beş aksiyomu sağlıyorsa matris normu olarak adlandırılır.

(1) $|\|A\|| \geq 0$,

(1a) Eğer $|\|A\||=0$ ise $A=0$ 'dır,

(2) $c \in \mathbb{C}$ için, $|\|c A\||=|c||\|A\||$,

(3) $|\|A+B\|| \leq|\|A\||+|\|B\||$,

(4) \|\|$A B\||\leq|\| A \| \mid$. $\|B\| \mid$.

Eğer $\forall A, B \epsilon \mathbb{C}^{n \times n}$ için (4) özelliği hariç diğer özellikler sağlanıyorsa bu durumda $|\|\|$.$| fonksiyonu$ genelleştirilmiş matris normu ya da vektör normu adını alır. Özel olarak bir $A \epsilon \mathbb{C}^{n \times n}$ matrisi için $\|A\|_{2}=$ $\left(\sum_{\mathrm{i}, \mathrm{j}=1}^{n}\left|a_{i j}\right|^{2}\right)^{\frac{1}{2}}$ şeklinde tanımlı norma Frobenius norm denir. Bir $A \in \mathbb{R}^{n \times n}$ matrisi için $K(A)=\|A\|_{2} \cdot\left\|A^{-1}\right\|_{2}$ ifadesi spektral koşul sayısı olarak tanımlanır.

Literatürdeki birçok çalışmada

$$
A x=b
$$

lineer denklem sisteminin ve bu sisteme dönüştürülebilen Lyapunov denklemleri ya da Sylvester matris denklemlerinin çözümlerini elde etmek için HSS, IHSS, MHSS, GMHSS vb. iteratif metotlar kullanılmıştır [örneğin, bakınız, 1,2,3]. M. Hajarian, genelleştirilmiş Sylvester transpoz matris denklem ikilisinin çözümünde BCR algoritması kullanmıştır[4]. Bu makalede [1] makalesinde verilen HSS metodundan esinlenerek Simetrik Ters Simetrik Ayrışım (SSS) metodunu ortaya koyacağız ve bu metot ile genelleştirilmiş Sylvester transpoz matris denklemi olan

$$
A X B+C X D+E X^{T} F=M
$$

matris denkleminin $X \in \mathbb{R}^{n \times n}$ çözümünü vereceğiz. Burada $A, B, C, D, E, F, M \in \mathbb{R}^{n \times n}$ bilinen matrislerdir. 


\section{II. ÖNBILGGILER}

Bu kısımda [1] çalışmasında ortaya konulan HSS iterasyonu tanıtılacaktır. Ayrıca, genelleştirilmiş Sylvester transpoz matris denkleminin kronecker çarpım yardımıyla (1) matris denklemi şeklinde nasıl yazılabileceği verilecektir.

\section{HSS iterasyon metodu.}

Verilen bir $x^{(0)}$ başlangıç vektörü için $\left\{x^{(k)}\right\}, k=0,1,2, \cdots$, dizisi

$$
\left\{\begin{array}{c}
(\alpha I+H) x^{\left(k+\frac{1}{2}\right)}=(\alpha I-S) x^{(k)}+b \\
(\alpha I+S) x^{(k+1)}=(\alpha I-H) x^{\left(k+\frac{1}{2}\right)}+b
\end{array}\right.
$$

hesaplamaları ile oluşsun. Bu işlemler $x^{(k)}$ aranan çözüme yeterince yaklaşıncaya kadar yapılsın. $\alpha$ burada pozitif bir sabittir [1]. Burada H ve S matrisleri Teorem l'deki gibidir.

Bu metodun yakınsaklığını gösteren ve [1]'de verilen teorem aşağıdaki gibidir.

Teorem 1. $A \in C^{n \times n}$ bir pozitif tanımlı matris olsun. Ayrıca $H=\frac{1}{2}\left(A+A^{*}\right)$ ve $S=\frac{1}{2}\left(A-A^{*}\right)$ onun hermityen ve ters hermityen kısımları ve $\alpha$ pozitif bir sabit olsun. Bu durumda HSS iterasyonunun $M(\alpha)$ iterasyon matrisi

$$
M(\alpha)=(\alpha I+S)^{-1}(\alpha I-H)(\alpha I+H)^{-1}(\alpha I-S)
$$

ile verilir. $M(\alpha)$ 'nın spektral yarıçapı $\rho(M(\alpha))$,

$$
\sigma(\alpha) \equiv \max _{\lambda_{i} \in \lambda(H)}\left|\frac{\alpha-\lambda_{i}}{\alpha+\lambda_{i}}\right|
$$

ile sınırlıdır. Burada, $\lambda(H), H$ matrisinin spektral kümesidir. Böylece $\forall \alpha>0$ için

$$
\rho(M(\alpha)) \leq \sigma(\alpha)<1
$$

sağlanır. Yani HSS iterasyonu $A x=b$ matris denkleminin $x^{*} \in \mathbb{C}^{n}$ yegane çözümüne yakınsar [1].

Şimdi de genelleştirilmiş Sylvester transpoz matris denklemini $A x=b$ matris denklemi ile ilişkilendireceğiz. (2) genelleştirilmiş Sylvester transpoz matris denklemi ele alınsın. Bu matris denklemi kronecker çarpım kullanılarak

$$
\left(B^{T} \otimes A+D^{T} \otimes \mathrm{C}+\left(F^{T} \otimes \mathrm{E}\right) P\right) \operatorname{vec}(x)=(\operatorname{vec}(M))
$$

şeklinde yazılabilir. Böylece,

$$
\begin{aligned}
& \mathcal{A}:=B^{T} \otimes A+D^{T} \otimes C+\left(F^{T} \otimes \mathrm{E}\right) P \text { ve } \mathcal{A} \in \mathbb{R}^{n^{2} \times n^{2}}, \\
& x:=\operatorname{vec}(X) \text { ve } x \in \mathbb{R}^{n^{2}}, \\
& b:=(\operatorname{vec}(M)) \text { ve } b \in \mathbb{R}^{n^{2}}
\end{aligned}
$$

olmak üzere genelleştirilmiş Sylvester transpoz matris denklemi $A x=b$ biçiminde yazılabilir. Buradaki $P$ matrisinin tanımı için aşağıdaki lemma gereklidir [7].

Lemma 1. $X \in \mathbb{R}^{n \times n}$ herhangi bir matris olsun. Bu durumda

$$
\operatorname{vec}\left(X^{T}\right)=\operatorname{Pvec}(X)
$$


olup burada $P$ matrisi, $n$ tamsayısı ile belirli, tek türlü tanımlı matristir. Ayrıca $P$ aşağıdaki özelliklere sahiptir.

i. Herhangi $n$ tamsayısı için $P \in \mathbb{R}^{n^{2} \times n^{2}}$ matrisi

$$
\left[\begin{array}{cccc}
E_{11}^{T} & E_{12}^{T} & \ldots & E_{1 n}^{T} \\
E_{21}^{T} & E_{22}^{T} & \ldots & E_{2 n}^{T} \\
\vdots & \vdots & \ddots & \vdots \\
E_{n 1}^{T} & E_{n 2}^{T} & \ldots & E_{n n}^{T}
\end{array}\right]
$$

biçimine sahiptir. Burada $E_{i j}, i, j=1,2, \ldots, n$ için $(i, j)$ elemanı 1 ve diğer elemanları 0 olan $n \times n$ boyutlu reel matristir.

ii. Herhangi $n$ tamsayısı için $P$ bir ortogonal matristir. Yani,

$$
P P^{T}=P^{T} P=I \text {. }
$$

\section{ANA SONUÇLAR}

Bu kısımda $A$ matrisinin simetrik - ters simetrik ayrışımı kullanılarak oluşturulan SSS metodu ortaya konulacaktır. Bu metodun yakınsaklığı gösterilip (2) denkleminin çözümü bu metot kullanılarak verilecektir.

Lemma 2. $A=M_{i}-N_{i}, i=1,2$, ifadeleri $A \in \mathbb{R}^{n \times n}$ matrisinin iki ayrışımı olsun ve $x^{(0)} \in \mathbb{R}^{n}$ bir verilmiş başlangıç vektörü olsun. Eğer $\left\{x^{(k)}\right\}$ iki kademeli iterasyon dizisi

$$
\left\{\begin{array}{c}
M_{1} x^{\left(k+\frac{1}{2}\right)}=N_{1} x^{(k)}+b, \\
M_{2} x^{(k+1)}=N_{2} x^{\left(k+\frac{1}{2}\right)}+b,
\end{array}\right.
$$

$k=0,1,2, \cdots$, ile tanımlanırsa bu durumda,

$$
x^{(k+1)}=M_{2}^{-1} N_{2} M_{1}^{-1} N_{1} x^{(k)}+M_{2}^{-1}\left(I+N_{2} M_{1}^{-1}\right) b
$$

elde edilir. Ayrıca $M_{2}^{-1} N_{2} M_{1}^{-1} N_{1}$ iterasyon matrisinin $\rho\left(M_{2}^{-1} N_{2} M_{1}^{-1} N_{1}\right)$ değeri, 1 'den küçükse bu durumda tüm $x^{(0)} \in \mathbb{R}^{n}$ başlangıç vektörleri için $\left\{x^{(k)}\right\}$ dizisi $A x=b$ sisteminin $x^{*} \in \mathbb{R}^{n}$ yegane çözümüne yakınsar.

Ispat.

$$
\begin{aligned}
& M_{1} x^{\left(k+\frac{1}{2}\right)}=N_{1} x^{(k)}+b \\
& M_{2} x^{(k+1)}=N_{2} x^{\left(k+\frac{1}{2}\right)}+b
\end{aligned}
$$

olsun. Bu durumda $M_{1}$ tersinir olduğundan (4) ifadesinden

$$
x^{\left(k+\frac{1}{2}\right)}=M_{1}^{-1} N_{1} x^{(k)}+M_{1}^{-1} b
$$

olur. (6) ifadesi (5) ifadesinde yerine yazılır ve $M_{2}$ matrisinin tersinir olduğu kullanılırsa

$$
x^{(k+1)}=M_{2}^{-1} N_{2} M_{1}^{-1} N_{1} x^{(k)}+M_{2}^{-1}\left(\mathrm{I}+N_{2} M_{1}^{-1}\right) b
$$

elde edilir 
$A=S+R$ olacak şekilde yazılsın. Burada $S$ simetrik, $R$ ters simetrik matrisleri $S=\frac{1}{2}\left(A+A^{T}\right)$ ve $R=$ $\frac{1}{2}\left(A-A^{T}\right)$ şeklinde belirtilsin. $\alpha \in \mathbb{R}^{+}$olmak üzere $A=(\alpha I+S)+(R-\alpha I)$ veya $A=(\alpha I+R)+(S-\alpha I)$ yazılabilir. Bu ifadeler $A x=b$ matris denkleminde yerine yazılırsa

$$
\begin{aligned}
& (\alpha I+S) x=(\alpha I-R) x+b \\
& (\alpha I+R) x=(\alpha I-S) x+b
\end{aligned}
$$

elde edilir. Yani (1) denklemi (7) veya (8) şeklinde de yazılabilir. Bu çalışma boyunca $S$ ve $R$ matrisleri yukarıdaki gibi olacaktır. Bu durumda SSS iterasyonu şu şekilde yazılır.

\section{SSS iterasyon metodu.}

$$
\begin{aligned}
& \text { Verilen bir } x^{(0)} \text { başlangıç vektörü için }\left\{x^{(k)}\right\}, k=0,1,2, \cdots, \text { dizisi } \\
& \left\{\begin{array}{c}
(\alpha I+S) x^{\left(k+\frac{1}{2}\right)}=(\alpha I-R) x^{(k)}+b \\
(\alpha I+R) x^{(k+1)}=(\alpha I-S) x^{\left(k+\frac{1}{2}\right)}+b
\end{array}\right.
\end{aligned}
$$

hesaplamaları ile oluşsun. Bu işlemler $x^{(k)}$ aranan çözüme yeterince yaklaşıncaya kadar yapılsın. a burada pozitif bir sabittir.

Teorem 2. $A \in \mathbb{R}^{n \times n}$ pozitif tanımlı bir matris ve $\alpha$ pozitif bir sabit olsun. Ayrıca $S=\frac{1}{2}\left(A+A^{T}\right)$ ve $R=$ $\frac{1}{2}\left(A-A^{T}\right)$ onun simetrik ve ters simetrik kısımları olsun. Bu durumda SSS iterasyonunun $M(\alpha)$ iterasyon matrisi;

$$
M(\alpha)=(\alpha I+R)^{-1}(\alpha I-S)(\alpha I+S)^{-1}(\alpha I-R)
$$

ile verilir. $M(\alpha)$ 'nın spektral yarıçapı $\rho(M(\alpha))$,

$$
\sigma(\alpha) \equiv \max _{\lambda_{i} \in \lambda(S)}\left|\frac{\alpha-\lambda_{i}}{\alpha+\lambda_{i}}\right|
$$

ile sinırlıdır. Burada $\lambda(S), S$ matrisinin spektral kümesidir. Böylece, $\forall \alpha>0$ için

$$
\rho(M(\alpha)) \leq \sigma(\alpha)<1
$$

olur. Yani SSS iterasyonu $A x=b$ denklem sisteminin $x^{*} \in \mathbb{R}^{n}$ yegane çözümüne yakınsar.

Ispat. Lemma 2' de verilen (3) ifadesinde

$$
M_{1}=(\alpha I+S), M_{2}=(\alpha I+R), N_{1}=(\alpha I-R), N_{2}=(\alpha I-S)
$$

alınırsa SSS metodunun iterasyon matrisi

$$
M(\alpha)=(\alpha I+R)^{-1}(\alpha I-S)(\alpha I+S)^{-1}(\alpha I-R)
$$

şeklinde bulunur. Burada $(\alpha I+S)$ ve $(\alpha I+R)$ yani $M_{1}$ ve $M_{2}$ tersinir kabul edilmiştir. Herhangi $A$ ve $B$ matrisleri için $\rho(A B)=\rho(B A)$ eşitliğinin varlığı bilindiğinden [6]

$$
\rho(M(\alpha))=\rho\left((\alpha I-S)(\alpha I+S)^{-1}(\alpha I-R)(\alpha I+R)^{-1}\right)
$$

yazilır. Ayrica,

$$
\rho(M(\alpha)) \leq\|M(\alpha)\|_{2}
$$


eşitsizliği bilindiğinden,

$$
\begin{aligned}
\rho(M(\alpha)) & \leq\left\|(\alpha I-S)(\alpha I+S)^{-1}(\alpha I-R)(\alpha I+R)^{-1}\right\|_{2} \\
\leq & \left\|(\alpha I-S)(\alpha I+S)^{-1}\right\|_{2} \cdot\left\|(\alpha I-R)(\alpha I+R)^{-1}\right\|_{2}
\end{aligned}
$$

yazılabilir. Burada

$$
Q(\alpha)=(\alpha I-R)(\alpha I+R)^{-1}
$$

alınırsa

$$
\begin{aligned}
Q(\alpha) \cdot Q^{T}(\alpha)=(\alpha I-R) & (\alpha I+R)^{-1}\left((\alpha I-R)(\alpha I+R)^{-1}\right)^{T} \\
& =(\alpha I-R)(\alpha I+R)^{-1}(\alpha I-R)^{-1}(\alpha I+R) \\
& =(\alpha I-R)((\alpha I-R)(\alpha I+R))^{-1}(\alpha I+R) \\
& =(\alpha I-R)\left(\alpha^{2} I-R^{2}\right)^{-1}(\alpha I+R) \\
& =(\alpha I-R)(\alpha I-R)^{-1}(\alpha I+R)^{-1}(\alpha I+R)=I
\end{aligned}
$$

olur. Yani $Q(\alpha)$ 'nın ortogonal matris olduğu görülür. Böylece $\|Q(\alpha)\|_{2}=1$ dir. Buradan,

$$
\rho(M(\alpha)) \leq\left\|(\alpha I-S)(\alpha I+S)^{-1}\right\|_{2}=\sigma(\alpha)
$$

olur. $\alpha$ bir pozitif sabit ve $\lambda_{i}>0, i=1,2, \cdots, n$, olduğundan

$$
\begin{aligned}
& \sigma(\alpha)=\max _{\lambda_{i} \in \lambda(S)}\left|\frac{\alpha-\lambda_{i}}{\alpha+\lambda_{i}}\right| \\
& \rho(M(\alpha)) \leq \sigma(\alpha)<1
\end{aligned}
$$

yazılabilir.

$\mathrm{Bu}$ teorem SSS iterasyonunun yakınsama hızının yalnızca $S$ kısmının spektrumuna bağlı olduğunu gösterir. $R$ kısmının spektrumuna, $A$ ya, ya da $S, R, A$ matrislerinin özvektörlerine bağlı değildir.

$x \in \mathbb{R}^{n}$ vektörü için bir vektör norm

$$
|\|x\||=\|(\alpha I+R) x\|_{2}
$$

şeklinde ve $\mathrm{X} \in \mathbb{R}^{n \times n}$ için bir matris norm \|\|$X\|=\|(\alpha I+R) X(\alpha I+R)^{-1} \|_{2}$ şeklinde verilirse teoremin ispatından,

$$
\|\| M(\alpha)\|\mid=\|(\alpha I-S)(\alpha I+S)^{-1}(\alpha I-R)(\alpha I+R)^{-1} \|_{2} \leq \sigma(\alpha)
$$

olduğu görülür. Buradan da

$$
\left|\left\|x^{(k+1)}-x^{*}\right\|\right| \leq \sigma(\alpha)\left|\left\|x^{(k)}-x^{*}\right\|\right|, k=0,1,2, \cdots,
$$

elde edilir. Böylece $\sigma(\alpha)$, |||. || normu anlamında SSS iterasyonunun küçülme katsayısının bir üst sınırıdır. $S$ 'nin maksimum ve minimum özdeğerleri biliniyorsa $\sigma(\alpha)$ için en iyi $\alpha$ parametresi $\rho(M(\alpha))$ veya $|\|M(\alpha)\||$ olur. Bu durum aşağıdaki sonuçta verilmiştir. 
Sonuç 1. $A \in \mathbb{R}^{n \times n}$ bir pozitif tanımlı matris, $S=\frac{1}{2}\left(A+A^{T}\right)$ ve $R=\frac{1}{2}\left(A-A^{T}\right)$ sirasıly onun simetrik ve ters simetrik kısımları ve $\gamma_{\min }, \gamma_{\max }$ sırasıyla $S$ 'nin en küçük ve en büyük özdeğerleri olsun. Ayrıca $\alpha$ pozitif bir sabit olsun. Bu durumda

$$
\alpha^{*}=\arg \min _{\alpha}\left\{\max _{\gamma_{\min } \leq \lambda \leq \gamma_{\max }}\left|\frac{\alpha-\lambda}{\alpha+\lambda}\right|\right\}=\sqrt{\gamma_{\min } \cdot \gamma_{\max }}
$$

ve

$$
\sigma\left(\alpha^{*}\right)=\frac{\sqrt{\gamma_{\max }}-\sqrt{\gamma_{\min }}}{\sqrt{\gamma_{\max }}+\sqrt{\gamma_{\min }}}=\frac{\sqrt{K(S)}-1}{\sqrt{K(S)}+1}
$$

olur. Burada $K(S), S$ matrisinin spektral koşul sayısıdır.

$$
\begin{gathered}
\text { Íspat. } \sigma(\alpha)=\max _{\lambda_{i} \in \lambda(S)}\left|\frac{\alpha-\lambda_{i}}{\alpha+\lambda_{i}}\right| \text { olduğu teoremden biliniyor. Buna göre } \\
\sigma(\alpha)=\max \left\{\left|\frac{\alpha-\gamma_{\min }}{\alpha+\gamma_{\min }}\right|,\left|\frac{\alpha-\gamma_{\max }}{\alpha+\gamma_{\max }}\right|\right\}
\end{gathered}
$$

olur. SSS iterasyonunun $\rho(M(\alpha))$ yakınsaklık çarpanını minimum yapacak şekilde bir yaklaşık optimal $\alpha>0$ değeri hesaplamak için $\rho(M(\alpha))$ yerine $\sigma(\alpha)$ üst sınırı minimize edilir. Eğer $\alpha^{*}$ böyle bir minimum nokta ise bu durumda $\alpha^{*}-\gamma_{\min }>0$ ve $\alpha^{*}-\gamma_{\max }<0$ ve

$$
\frac{\alpha^{*}-\gamma_{\min }}{\alpha^{*}+\gamma_{\min }}=\frac{\gamma_{\max }-\alpha^{*}}{\gamma_{\max }+\alpha^{*}}
$$

sağlamak zorundadır. Böylece,

$$
\alpha^{*}=\sqrt{\gamma_{\min } \gamma_{\max }}
$$

bulunur. $\alpha^{*}$ 'n bu değeri $\sigma(\alpha)=\max \left\{\left|\frac{\alpha-\gamma_{\min }}{\alpha+\gamma_{\min }}\right|,\left|\frac{\alpha-\gamma_{\max }}{\alpha+\gamma_{\max }}\right|\right\}$ ifadesinde yerine yazıllırsa

$$
\begin{aligned}
& \sigma\left(\alpha^{*}\right)=\max \left\{\left|\frac{\alpha^{*}-\gamma_{\min }}{\alpha^{*}+\gamma_{\min }}\right|,\left|\frac{\alpha^{*}-\gamma_{\max }}{\alpha^{*}+\gamma_{\max }}\right|\right\} \\
& \sigma\left(\alpha^{*}\right)=\max \left\{\left|\frac{\sqrt{\gamma_{\max }}-\sqrt{\gamma_{\min }}}{\sqrt{\gamma_{\max }}+\sqrt{\gamma_{\min }}}\right|,\left|\frac{\sqrt{\gamma_{\min }}-\sqrt{\gamma_{\max }}}{\sqrt{\gamma_{\min }}+\sqrt{\gamma_{\max }}}\right|\right\}
\end{aligned}
$$

elde edilir ve ispat tamamlanır.

Tüm bu yapılanlara göre artık aşağıdaki sonuç verilebilir.

Teorem 3. $A, B, C, D, E, F, M \in \mathbb{R}^{n \times n}$, bilinen matrisler, $X \in \mathbb{R}^{n \times n}$ bilinmeyenler matrisi olmak üzere, $A X B+C X D+E X^{T} F=M$

genelleştirilmiş Sylvester transpoz matris denklemi

$$
\begin{aligned}
& A:=B^{T} \otimes A+D^{T} \otimes C+\left(F^{T} \otimes \mathrm{E}\right) P \text { ve } A \in \mathbb{R}^{n n \times n n}, \\
& x:=\operatorname{vec}(X) \text { ve } x \in \mathbb{R}^{n^{2}} \\
& b:=(\operatorname{vec}(M)) \text { ve } b \in \mathbb{R}^{n^{2}}
\end{aligned}
$$


şeklinde düzenleme ile $A x=b$ biçiminde yazılır. Bu durumda $\alpha^{*}=\sqrt{\gamma_{\min } \cdot \gamma_{\max }}, S=\frac{1}{2}\left(A+A^{T}\right)$ ve $R=$ $\frac{1}{2}\left(A-A^{T}\right)$ olmak üzere

$$
x^{(k+1)}=(\alpha I+R)^{-1}(\alpha I-S)(\alpha I+S)^{-1}(\alpha I-R) x^{(k)}+(\alpha I+R)^{-1}\left((\alpha I-S)(\alpha I+S)^{-1}+I\right) b
$$

ile bulunan $x$ vektörü gerçek çözüm $x^{*}$ iken, $\operatorname{vec}\left(x^{*}\right) \in \mathbb{R}^{n^{2}}$ vektörüne yakınsar.

$\mathrm{Bu}$ teoreme göre

$$
A X B+C X D+E X^{T} F=M
$$

genelleştirilmiş Sylvester transpoz matris denkleminin $X \in \mathbb{R}^{n \times n}$ çözümünü bulan algoritma aşağıdaki gibi yazılabilir.

\section{Algoritma.}

Adım 1. $n$ ve $d$ gir. Burada $n$, matrislerin boyutu ve $d, A X B+C X D+E X^{T} F-M$ matrisinin normu için istenen üst sinırdır.

Adim 2. $A, B, C, D, E, F, M, X_{0} \in \mathbb{R}^{n \times n}$ gir. $k=0$ olsun.

Adım 3. $P=\left[E_{i j}\right]_{n^{2} \times n^{2}}$ matrisini oluştur,

Adım 4. $A:=B^{T} \otimes A+D^{T} \otimes C+\left(F^{T} \otimes \mathrm{E}\right) P$ matrisini ve $b:=\operatorname{vec}(M)$ ve $x^{(k)}=\operatorname{vec}\left(X_{0}\right)$ vektörlerini oluştur,

Adım 5. A matrisinin öz değerlerinde negatif veya kompleks eleman varsa 'Adım 1.' e git.

Adim 6. $S=\frac{1}{2}\left(\mathcal{A}+A^{T}\right), R=\frac{1}{2}\left(\mathcal{A}-\mathcal{A}^{T}\right)$ olarak hesapla,

Adım 7. $\alpha=\sqrt{\gamma_{\min } \cdot \gamma_{\max }}$ olarak hesapla,

Adim 8. $x^{(k+1 / 2)}=(\alpha I+S)^{-1}(\alpha I-R) x^{(k)}+(\alpha I+S)^{-1} b$ ve

$$
x^{(k+1)}=(\alpha I+R)^{-1}(\alpha I-S) x^{(k+1 / 2)}+(\alpha I+R)^{-1} b \text { olarak hesapla, }
$$

Adım 9. $x^{(k+1)}=\operatorname{vec}(X)$ olacak şekilde $X$ matrisini oluştur,

Adım 10. $\left\|A X B+C X D+E X^{T} F-M\right\|_{2}<d$ ise dur, değilse 'Adım 8.' e git.

Şimdi bu teoremin etkinliğini gösteren bir örnek verilecektir.

Örnek.

$$
\begin{gathered}
A=\left(\begin{array}{ccc}
10 & 6 & 5 \\
6 & 8 & 5 \\
5 & 5 & 10
\end{array}\right), B=\left(\begin{array}{ccc}
10 & 5 & 6 \\
5 & 10 & 5 \\
6 & 5 & 8
\end{array}\right), C=\left(\begin{array}{ccc}
10 & 4 & 5 \\
4 & 4 & 3 \\
5 & 3 & 6
\end{array}\right), D=\left(\begin{array}{ccc}
10 & 5 & 2 \\
5 & 6 & 6 \\
2 & 6 & 8
\end{array}\right), \\
E=\left(\begin{array}{lll}
0 & 4 & 4 \\
4 & 0 & 3 \\
4 & 3 & 4
\end{array}\right), F=\left(\begin{array}{ccc}
0 & 3 & 5 \\
3 & 2 & 6 \\
5 & 6 & 10
\end{array}\right), M=\left(\begin{array}{ccc}
8 & 1 & 9 \\
1 & 8 & 5 \\
9 & 5 & 10
\end{array}\right)
\end{gathered}
$$

verilen matrisleri ve 


$$
X_{0}=\left(\begin{array}{ccc}
10 & 11 & 10 \\
11 & 6 & 9 \\
10 & 9 & 12
\end{array}\right)
$$

seçilen matrisi için

$$
A X B+C X D+E X^{T} F=M
$$

genelleştirilmiş Sylvester transpoz matris denkleminin $X \in \mathbb{R}^{3 \times 3}$ çözümü SSS metodu ile 28 adımda

$$
X=\left(\begin{array}{ccc}
0,0707 & -0,1841 & 0,1481 \\
-0,1120 & 0,2456 & -0,1630 \\
0,0702 & -0,0774 & 0,0658
\end{array}\right)
$$

olarak elde edilir. Burada $\left\|A X B+C X D+E X^{T} F-M\right\|=0,0854$ olur.

\section{KAYNAKLAR}

[1] Z.Z. Bai, G.H. Golub and M.K. Ng (2003). Hermitian and Skew Hermitian Splitting Methods for NonHermitian Positive Definitive Linear Systems, SIAM J. Appl. Math. 24(3), 603-626.

[2] Z.Z. Bai, M. Benzi, F. Chen (2011). On preconditioned MHSS iteration methods for complex symmetric linear systems, Numer. Algorithms, 56, 297-317.

[3] M. Dehghan, A. Shirilord (2019). A generalized modified Hermitian and Skew Hermitian splitting (GMHSS) method for solving complex Sylvester matrix equation, Appl. Math. Comput. 348, 632-651.

[4] M. Hajarian (2018). Biconjugate residual algorithm for solving General Sylvester-transpose matrix equations, Filomat, 32:15, 5307-5318.

[5] J.W. Demmel, Applied Numerical Linear Algebra (first edition), Siam, Berkeley (1997).

[6] R.A. Horn, C.R. Johnson, Matrix Analysis (second edition), Cambridge University Press, New York (2012).

[7] B. Zhou, J. Lam, G.R. Duan (2011). Toward solution of matrix equation $X=\operatorname{Af}(X) B+C$, Linear Algebra Appl. 435, 1370-1398. 